\title{
CONVEX ANALYSIS AND THERMODYNAMICS
}

\author{
Nelly Point \\ Université Paris-Est/Laboratoire Navier \\ Ecole des Ponts ParisTech \\ 6 et 8 av. Blaise Pascal, Cité Descartes, Champs-sur-Marne \\ 77455 Marne-la-Vallée, Cedex 2, France \\ Silvano ERLICHER \\ EGIS Industries, Direction Technique et Scientifique \\ 4, rue Dolores Ibarruri \\ 93188 Montreuil Cedex, France
}

\begin{abstract}
Convex analysis is very useful to prove that a material model fulfills the second law of thermodynamics. Dissipation must remains non-negative and an elegant way to ensure this property is to construct an appropriate pseudo-potential of dissipation. In such a case, the corresponding material is said to be a Standard Generalized Material and the flow rules fulfill a normality rule (i.e. the dissipative thermodynamic forces are assumed to belong to an admissible domain and the flow of the corresponding state variables is orthogonal to the boundary of this domain). The sum of the pseudo-potential with its Legendre-Fenchel conjugate fulfills the Fenchel's inequality and as the actual value of the dual pair forces-flows minimizes this inequality, this can be used as a convergence criterium for numerical applications. Actually, some very commonly used and effective models do not fit into that family of Standard Generalized Materials. A procedure is here proposed which permits to retrieve the normality assumption and to construct a pair of dual pseudo-potentials also for these non-standard material models. This procedure was first presented by the authors for non-associated plasticity. Now it is extended to a large range of mechanical problems.
\end{abstract}

Why such a subject in a book in memory of Professor Seiji Ukaï ?

A long time ago, as he was invited in Paris for one year, he gave a course in French on Boltzman equation. My friend Hamid Ghidouche and myself attended his course. We had the chance to work and to publish our first article with Professor Ukaï. Then, even if we get more interested in mechanical problems, since that time we always kept in touch. He was a great mathematician and a very kind person. It is a great honor to participate to this book in his memory.

1. Introduction. Convex analysis is very useful to prove that a rate-independent material model fulfills the second law of thermodynamics. Dissipation must remains non-negative and an elegant way to obtain this is to construct a suitable convex pseudo-potential of dissipation [1]. In such a case, the corresponding material is said to be a Standard Generalized Material [2] and the constitutive law fulfills a normality rule (i.e. the dissipative thermodynamic forces are assumed to belong

2010 Mathematics Subject Classification. Primary: 74C05, 74A15, 74A20.

Key words and phrases. Convex analysis, Thermodynamics, Non-associated flow rules, Pseudopotentials, Legendre-Fenchel conjugate, Dissipation. 
to an admissible domain and the flow of the corresponding state variables must be orthogonal to the boundary of this domain). In this case, the flow rule for the state variables is called 'associated'. Moreover, the sum of the pseudo-potential of dissipation with its Legendre-Fenchel conjugate, fulfills the Fenchel's inequality and the physical or actual values of the dual pair forces-flows minimizes the inequality. This minimization property can be used as a convergence criterium for numerical applications.

Actually, some very commonly used and effective models are characterized by non-associated flow rules. Hence, they do not fit into the family of Standard Generalized Materials. However, we have already shown that in some particular cases it is possible to find suitable pseudo-potentials of dissipation allowing the normality property to be retrieved, provided that these potentials have a parametric dependence on the material state [3], [4], [5]. A procedure is here proposed which permits to construct a pair of such convex dissipation potentials for a very large class of mechanical problems and to retrieve the normality assumption.

This procedure was first presented for non-associated plasticity during a congress in 2010. Then, it was generalized to the case of unilateral contact with friction [6]. Now it is extended to a large range of constitutive laws. By the authors' knowledge, very few other mathematical analyses exist for non-standard materials, e.g. [7], [8].

This presentation is structured as follows. In the first part, useful and classical tools of convex analysis are recalled. Then, in the second part, thermodynamics and its link with convex analysis is presented as well as the concept of Standard Generalized Materials and its relationship with the convex functions called dissipation potentials and with the normality rule. In the third part, a constructive procedure is proposed to define state-dependent pseudo-potentials for constitutive laws traditionally considered as 'non-associated'. The main idea is to accept to consider a state-dependent admissible domain for the dissipative thermodynamic forces, in order to retrieve the normality rule for the flow of the state variables.

2. Convex analysis. In this section, some classical but essential notions involving duality and the Legendre-Fenchel transform are recalled. They can be found in the fundamental work of Rockafellar [9].

2.1. Legendre-Fenchel conjugates and Fenchel inequality. Let $\mathbb{V}$ and $\mathbb{V}^{*}$ be two dual vector spaces associated through a bilinear form (denoted by a dot):

$$
\begin{aligned}
\mathbf{x}, \mathbf{x}^{*} & \rightarrow \mathbf{x}^{*} \cdot \mathbf{x} \\
\mathbb{V} \times \mathbb{V}^{*} & \rightarrow \mathbb{R}
\end{aligned}
$$

with $\mathbb{R}$ the set of real numbers. Moreover, let $\phi(\mathbf{x})$ be a proper convex function from $\mathbb{V}$ to $]-\infty,+\infty]$. By definition, the effective domain of $\phi$ reads

$$
\operatorname{dom} \phi=\{\mathbf{x} \in \mathbb{V} / \quad \phi(\mathbf{x})<+\infty\}
$$

If $\phi$ is convex and differentiable on $\mathbb{V}$, then the gradient of $\phi$ in $\mathbf{x} \in$ dom $\phi$, i.e. $\nabla \phi(\mathbf{x}) \in \mathbb{V}^{*}$, fulfills the following condition:

$$
\forall \mathbf{y} \in \operatorname{dom} \phi, \quad \phi(\mathbf{y})-\phi(\mathbf{x}) \geq \nabla \phi(\mathbf{x}) \cdot(\mathbf{y}-\mathbf{x})
$$

If $\phi$ is convex and not differentiable, this notion has to be generalized: $\mathbf{x}^{*}$ belonging to $\mathbb{V}^{*}$ is called a subgradient of $\phi$ in $\mathbf{x} \in \operatorname{dom} \phi$ if

$$
\forall \mathbf{y} \in \operatorname{dom} \phi, \quad \phi(\mathbf{y})-\phi(\mathbf{x}) \geq \mathbf{x}^{*} \cdot(\mathbf{y}-\mathbf{x})
$$


The subdifferential in $\mathbf{x}, \partial \phi(\mathbf{x})$, is the set of all the $\mathbf{x}^{*}$ fulfilling (1). When $\partial \phi(\mathbf{x})$ is non-empty, $\phi$ is said subdifferentiable in $\mathbf{x}$.

The relationship in (1) can be rewritten as follows:

$$
\forall \mathbf{y} \in \operatorname{dom} \phi, \quad \phi(\mathbf{x})+\mathbf{x}^{*} \cdot \mathbf{y}-\phi(\mathbf{y}) \leq \mathbf{x}^{*} \cdot \mathbf{x}
$$

Hence, it is natural to introduce the Legendre-Fenchel conjugate of $\phi$ defined by:

$$
\phi^{*}\left(\mathbf{x}^{*}\right)=\sup _{\mathbf{y} \in \mathbb{V}}\left(\mathbf{x}^{*} \cdot \mathbf{y}-\phi(\mathbf{y})\right)
$$

This function $\phi^{*}$ is always convex and when $\phi$ is convex, one also has $\phi^{* *}=\phi$. If $\phi$ is not convex, it is possible to define $\phi^{*}$ and $\phi^{* *}$, but in this case $\phi^{* *}$ is the closure of the convexified function $\phi$, i.e. $\phi^{* *}=c l(\operatorname{conv} \phi)$.

Due to the definition of $\phi^{*}$ in (2), the following inequality, called Fenchel inequality, is always verified:

$$
\forall\left(\mathbf{x}, \mathbf{x}^{*}\right) \in \mathbb{V} \times \mathbb{V}^{*} \quad \phi(\mathbf{x})+\phi^{*}\left(\mathbf{x}^{*}\right) \geq \mathbf{x}^{*} \cdot \mathbf{x}
$$

The following proposition is very useful for applications in mechanics.

Proposition 1. For a function $\phi$, convex, proper, lower semi-continuous from $\mathbb{V}$ to ]$-\infty,+\infty]$ and for a couple $\left(\tilde{\mathbf{x}}, \tilde{\mathbf{x}}^{*}\right)$ the following three relationships are equivalent:

1. $\phi(\tilde{\mathbf{x}})+\phi^{*}\left(\tilde{\mathbf{x}}^{*}\right)=\tilde{\mathbf{x}}^{*} \cdot \tilde{\mathbf{x}}$

2. $\tilde{\mathbf{x}} \in \partial \phi^{*}\left(\tilde{\mathbf{x}}^{*}\right)$

3. $\tilde{\mathbf{x}}^{*} \in \partial \phi(\tilde{\mathbf{x}})$

The lemma hereafter will be used later.

Lemma 1. For a function $\phi: \mathbb{V} \rightarrow]-\infty, \infty]$ convex, proper, non-negative and such that $\phi(\mathbf{0})=0$, if $\mathbf{x}^{*}$ belongs to the subdifferential of $\phi$ in $\mathbf{x}$, then $\mathbf{x}^{*} \cdot \mathbf{x}$ is positif:

$$
\mathbf{x}^{*} \in \partial \phi(\mathbf{x}) \quad \Longrightarrow \quad \mathbf{x}^{*} \cdot \mathbf{x} \geq 0
$$

2.2. Indicator function. The indicator function of a domain $\mathbb{E} \subset \mathbb{V}^{*}$ is defined as follows:

$$
\mathbb{I}_{\mathbb{E}}\left(\mathbf{x}^{*}\right):=\left\{\begin{array}{cc}
0 & \text { for } \mathbf{x}^{*} \in \mathbb{E} \\
+\infty & \text { for } \mathbf{x}^{*} \notin \mathbb{E}
\end{array}\right.
$$

The function $\mathbb{I}_{\mathbb{E}}\left(\mathbf{x}^{*}\right)$ is convex, proper, lower semi-continuous non-negative and such that $\mathbb{I}_{\mathbb{E}}(\mathbf{0})=0$ if the domain $\mathbb{E}$ is convex closed and contains $\mathbf{0}$. In this case, the Lemma 1 applies and $\mathbf{x} \in \partial \mathbb{I}_{\mathbb{E}}\left(\mathbf{x}^{*}\right)$ implies $\mathbf{x}^{*} \cdot \mathbf{x} \geq 0$.

The Legendre-Fenchel conjugate function $\mathbb{I}_{\mathbb{E}}^{*}(\mathbf{x})$ is defined by:

$$
\mathbb{I}_{\mathbb{E}}^{*}(\mathbf{x}):=\sup _{\mathbf{x}^{*} \in \mathbb{E}}\left(\mathbf{x}^{*} \cdot \mathbf{x}\right)
$$

and is positively homogeneous of degree one. If the function $\mathbb{I}_{\mathbb{E}}^{*}(\mathbf{x})$ is denoted by $\phi(\mathbf{x})$, the Legendre-Fenchel conjugate $\phi^{*}\left(\mathbf{x}^{*}\right)$ is equal to $\mathbb{I}_{\mathbb{E}}^{* *}\left(\mathbf{x}^{*}\right)=\mathbb{I}_{\mathbb{E}}\left(\mathbf{x}^{*}\right)$, since $\mathbb{E}$ is closed and convex.

For a couple $\left(\mathbf{x}, \mathbf{x}^{*}\right)$ realizing the minimum of the sum $\phi(\mathbf{x})+\phi^{*}\left(\mathbf{x}^{*}\right)$, Proposition 1 and the given definitions of $\phi(\mathbf{x})$ and $\phi^{*}\left(\mathbf{x}^{*}\right)$ imply the following equivalences:

$$
\left\{\mathbf{x}^{*} \in \mathbb{E} \text { and } \phi(\mathbf{x})=\mathbf{x}^{*} \cdot \mathbf{x}\right\} \quad \Longleftrightarrow \mathbf{x}^{*} \in \partial \phi(\mathbf{x}) \quad \Longleftrightarrow \mathbf{x} \in \partial \phi^{*}\left(\mathbf{x}^{*}\right)
$$


If the domain $\mathbb{E}$ is defined by a function $f$ such that:

$$
\mathbb{E}:=\left\{\mathbf{x}^{*} \in \mathbb{V} / \quad f\left(\mathbf{x}^{*}\right) \leq 0 \quad \text { with } \quad f(\mathbf{0})=0\right\}
$$

and if $f$ is convex and differentiable, then for $\mathbb{I}_{\mathbb{E}}\left(\mathbf{x}^{*}\right)$, since the domain $\mathbb{E}$ is not empty, the following equivalence holds true:

$$
\mathbf{x} \in \partial \mathbb{I}_{\mathbb{E}}\left(\mathbf{x}^{*}\right) \Longleftrightarrow\left\{\begin{array}{c}
\mathbf{x}=\lambda \nabla f\left(\mathbf{x}^{*}\right) \\
f\left(\mathbf{x}^{*}\right) \leq 0, \quad \lambda \geq 0, \quad \lambda f\left(\mathbf{x}^{*}\right)=0
\end{array}\right.
$$

This implies that, on the boundary of $\mathbb{E}$, the elements of the subgradient of $\mathbb{I}_{\mathbb{E}}$ are normal to this boundary. By the Proposition 1, this is equivalent to $\mathbf{x}^{*} \in \partial \mathbb{I}_{\mathbb{E}}^{*}(\mathbf{x})$ and is generally referred to as the normality rule. It implies $\mathbf{x}^{*} \cdot \mathbf{x} \geq 0$.

3. Thermodynamics. In this section, we assume small deformations and isothermal transformations and, for the sake of simplicity, we will assume that the state of the material can be defined by the strain tensor $\varepsilon$ and a vector $\alpha$ containing all the internal variables (i.e. the plastic strain tensor, the damage, etc). In this case, the associated thermodynamic forces are respectively $\sigma$, the stress tensor, and $A$, the thermodynamic forces associated with the internal variables.

3.1. Dissipative and non-dissipative forces. In the case of isothermal processes, the second principle of thermodynamics, together with the first principle, entails that a potential $\psi(\varepsilon, \alpha)$ must exist such that the quantity

$$
D:=\sigma: \dot{\varepsilon}-\dot{\psi}(\varepsilon, \alpha)
$$

is non-negative. $D \geq 0$ is the energy dissipation rate per unit volume; : indicates the double-dot product (double contraction) of two second order tensors; the function $\psi(\varepsilon, \alpha)$ is named Helmoltz free energy; the superposed dot indicates the time-differentiation.

The non-dissipative thermodynamic forces (or reversible forces [10], or quasiconservative forces [11]) are defined as the gradient of the free energy density $\psi(\varepsilon, \alpha)$ :

$$
\begin{aligned}
\sigma^{n d} & =\frac{\partial \psi}{\partial \varepsilon} \\
A^{n d} & =\frac{\partial \psi}{\partial \alpha}
\end{aligned}
$$

Thus, the dissipation can be written as:

$$
D=\sigma: \dot{\varepsilon}-\sigma^{n d}: \dot{\varepsilon}-A^{n d} \cdot \dot{\alpha}
$$

On the other hand, starting from the observation that the dissipation $D$ is associated with the evolution of the internal variables and/or the strain $\varepsilon$, it is natural to postulate the following expression, alternative to Eq. (12):

$$
D:=\sigma^{d}: \dot{\varepsilon}+A^{d} \cdot \dot{\alpha}
$$

where $\left(\sigma^{d}, A^{d}\right):=\mathbf{f}^{d}$ are the so-called dissipative thermodynamics forces, $\sigma^{d}$ being associated with $\varepsilon$ and $A^{d}$ with the internal variables $\alpha$. An effective method for defining the forces $\mathbf{f}^{d}$, in such a way that the non-negativity of $D$ is guaranteed, is based on the use of the dissipation potential, also called pseudo-potential; see the next sections.

Eqs. (12) and (13) represent the same physical quantity. Hence, they must be equal for any flow $(\dot{\varepsilon}, \dot{\alpha})$. This implies

$$
\begin{gathered}
\sigma=\sigma^{n d}+\sigma^{d} \\
A:=A^{n d}+A^{d}=0
\end{gathered}
$$


In a more compact form, one can write

$$
\mathbf{f}:=(\sigma, A)=\left(\sigma^{n d}+\sigma^{d}, 0\right)=\left(\sigma^{n d}, A^{n d}\right)+\left(\sigma^{d}, A^{d}\right):=\mathbf{f}^{n d}+\mathbf{f}^{d}
$$

Hence, all the thermodynamic forces are decomposed into a non-dissipative and a dissipative part [12]. The sum $A$ of the forces associated with the internal variables is always equal to 0.

If it is assumed that there is no viscous dissipation (i.e. no term depending on $\dot{\varepsilon}$ in the dissipation D), then the term $\sigma^{d}$ should remain equal to zero. Hence, in this special case relationships (14) become:

$$
\begin{gathered}
\sigma=\sigma^{n d} \\
0=A^{n d}+A^{d}
\end{gathered}
$$

and the dissipation reads:

$$
D=A^{d} \cdot \dot{\alpha}=-A^{n d} \cdot \dot{\alpha}
$$

It is interesting to remark that in classical treatises on continuum mechanics, e.g. [13], the distinction between non-dissipative and dissipative forces is not introduced and the relation (13) is written as follows:

$$
D:=-A \cdot \dot{\alpha}
$$

where $A$, here stands for the non-dissipative forces associated to the internal variables. Nonetheless, this distinction will be of great importance in the construction of state-dependent pseudo-potentials as it will appear later (see Section 5, in particular the definition of the state-dependent loading domain for non-associated flow rules given by Eq. (24)).

In the following subsection, the concepts of dissipation potentials (or pseudopotentials), proposed and used in [1] and [2], are introduced. They lead to an effective framework to study the dissipation in mechanics.

3.2. Associative plasticity. A plasticity model is said associative (or associated) when the thermodynamic force $A^{d}$ belongs to an elastic domain $\mathbb{E}$ defined by:

$$
\mathbb{E}:=\left\{A^{d} \in \mathbb{V}^{*} / \quad f\left(A^{d}\right) \leq 0 \quad \text { with } \quad f(\mathbf{0})=0\right\}
$$

with $f$ convex, differentiable, and if the flow of the internal variable $\dot{\alpha}$ is such that:

$$
\left\{\begin{array}{c}
\dot{\alpha}=\lambda \nabla f\left(A^{d}\right) \\
f\left(A^{d}\right) \leq 0, \quad \lambda \geq 0, \quad \lambda f\left(A^{d}\right)=0
\end{array}\right.
$$

Eq. (19) can be rewritten as follows, using Eq. (9):

$$
\dot{\alpha} \in \partial \mathbb{I}_{\mathbb{E}}\left(A^{d}\right)
$$

This normality assumption permits to define a pseudo-potential $\mathbb{I}_{\mathbb{E}}\left(A^{d}\right)$ as the indicator function of the elastic domain and to deduce that the conjugate function:

$$
\mathbb{I}_{\mathbb{E}}^{*}(\dot{\alpha}):=\sup _{A^{d^{\prime}} \in \mathbb{E}}\left(A^{d^{\prime}} \cdot \dot{\alpha}\right)
$$

is positively homogeneous of degree one. Here, as usually in mechanics, the prime stands for the generic variable and $A^{d}$ without a prime for the actual (physical) value. Introducing $\phi\left(\dot{\alpha}^{\prime}\right):=\mathbb{I}_{\mathbb{E}}^{*}\left(\dot{\alpha}^{\prime}\right)$, then $\phi^{*}\left(A^{d^{\prime}}\right):=\mathbb{I}_{\mathbb{E}}\left(A^{d^{\prime}}\right)$ and Fenchel's inequality is written as:

$$
\phi\left(\dot{\alpha}^{\prime}\right)+\phi^{*}\left(A^{d \prime}\right) \geq A^{d \prime} \cdot \dot{\alpha}^{\prime}
$$


The Proposition 1 implies that equality is reached for the actual couple $A^{d}$ and $\dot{\alpha}$. Using (7), the pseudo-potential of dissipation is such that:

$$
\phi(\dot{\alpha}):=A^{d} \cdot \dot{\alpha}
$$

which is non-negative, as it has been proven by Lemma 1 in the previous section. Hence, the second law is fulfilled. Moreover the dissipative forces are defined by:

$$
A^{d} \in \partial \phi(\dot{\alpha})
$$

In this case, the constitutive law is said to be associated, since the direction of the flow defined by (19) depends on the function $f$, which also defines the elastic domain and which is called the yield function.

3.3. Non-associative plasticity. Some well-known plasticity models do not enter in the framework of associative plasticity, because the direction of the flow $\dot{\alpha}$ is not defined by $\nabla f$ as in (19). Then, another function $g$, called the plastic potential, must be introduced to define the direction of the flow $\dot{\alpha}$ :

$$
\dot{\alpha}=\lambda \nabla g\left(A^{d}\right)
$$

with $f$ defining the elastic domain. In this case, the flow is not normal to the boundary of the elastic domain. Such models of plasticity are said non-associated.

4. More general associated and non-associated models. The concepts introduced in plasticity have been generalized and applied to other type of mechanical problems like unilateral contact with friction, damage, adhesion, etc. The observable variables are not restricted to be the strain tensor $\varepsilon$. For instance, they are the normal and tangential displacements in the case of unilateral contact with friction. The internal variable $\alpha$ can include irreversible displacements (e.g. sliding) in the case of unilateral contact with friction or the damage, the adhesion, etc. The same ideas as in the case of associative plasticity lead to the concept of Standard Generalized Materials [2], which is briefly recalled hereafter.

Let $\mathbf{v} \in \mathbb{V}$ be the vector of all the state variables (observable and internal). The corresponding vector of flows is $\dot{\mathbf{v}}$. The dual space $\mathbb{V}^{*}$ contains the so-called thermodynamic forces and the dissipative ones $\mathbf{f}^{d}$. If there exists a pseudo-potential $\phi(\dot{\mathbf{v}})$ convex, proper, and non-negative and such that $\phi(\mathbf{0})=0$, then using the Lemma 1 , the normality condition $\mathbf{f}^{d} \in \partial \phi(\dot{\mathbf{v}})$ entails the positivity of the dissipation:

$$
D=\mathbf{f}^{d} \cdot \dot{\mathbf{v}} \geq 0
$$

and conversely the flow fulfills $\dot{\mathbf{v}} \in \partial \phi^{*}\left(\mathbf{f}^{d}\right)$.

The constitutive law is said associated and the material of standard generalized type. However, a lot of materials need to be modelled with non-associated constitutive laws as, for instance, soils, rock, concrete, etc. Actually, in the case of a non-associated flow rule, it is not possible to construct pseudo-potentials of the form $\phi(\dot{\mathbf{v}})$ and $\phi^{*}\left(\mathbf{f}^{d}\right)$ (or $\phi(\dot{\mathbf{v}} ; \mathbf{v})$ and $\left.\phi^{*}\left(\mathbf{f}^{d} ; \mathbf{v}\right)\right)$. Nonetheless, it is possible to construct state-dependent conjugated pseudo-potentials of the form $\phi\left(\dot{\mathbf{v}} ; \mathbf{f}-\mathbf{f}^{n d}(\mathbf{v})\right)$ and $\phi^{*}\left(\mathbf{f}^{d} ; \mathbf{f}-\mathbf{f}^{n d}(\mathbf{v})\right)\left(\right.$ or $\phi\left(\dot{\mathbf{v}} ; \mathbf{f}-\mathbf{f}^{n d}(\mathbf{v}) ; \mathbf{v}\right)$ and $\left.\phi^{*}\left(\mathbf{f}^{d} ; \mathbf{f}-\mathbf{f}^{n d}(\mathbf{v}) ; \mathbf{v}\right)\right)$. In order to simplify the notation, and only for this reason, the possible direct dependence of $\phi$ and $\phi^{*}$ on the state variables $\mathbf{v}$ will not be explicity indicated hereinafter.

We have already succeeded to find such state-dependent pseudo-potentials in some particular cases [3], [4], [5], but without following a structured method. Then, we have proposed a constructive procedure in the case of plasticity and now we are able to extend it to more general cases. The assumptions are: 
- There exists a convex domain $\mathbb{E}$ for the admissible dissipative thermodynamic forces $\mathbf{f}^{d}$

- The flow rules are defined by a potential $g\left(\mathbf{f}^{d}\right)$ (eventually, $\nabla g$ non-normal to the boundary of the admissible domain $\mathbb{E}$ )

The main idea is to relax the constraints on the domain of the admissible dissipative thermodynamic forces $\mathbf{f}^{d}$ and to make it state-dependent, in order that the normality rule holds true. The procedure is presented hereinafter.

5. Construction of state-dependent pseudo-potentials. As it has been said before, the non-dissipative thermodynamic forces $\mathbf{f}^{n d}$ are defined as the gradient of the Helmholtz free density of energy. The sum $\mathbf{f}^{d}+\mathbf{f}^{n d}$ is equal to $\mathbf{f}$. For instance, in the case of plasticity, one has $\mathbf{f}=(\sigma, A)$, while in the case of unilateral contact $\mathbf{f}=(\mathbf{R}, A)$ with $\mathbf{R}$ the reaction reaction force. In both cases, $A$ defined in Eq. (14) as the sum of the dissipative and non dissipative thermodynamic forces associated to the internal variables is equal to 0 . At any time, $\mathbf{f}$ is supposed known: we recall that $\sigma$ and $\mathbf{R}$ must fulfil the dynamic or static equilibrium equation. The state variables $\mathbf{v}$ are also supposed known at any time. Hence, the value of the nondissipative thermodynamic forces $\mathbf{f}^{\text {nd }}$ can be calculated knowing the free energy $\psi=\psi(\mathbf{v})$ and the values of the state variables.

In the next subsections, assuming that at any time the evolution of the system depends on the values of the total forces $\mathbf{f}$ and on the state variables $\mathbf{v}$, we intend to show how to construct a couple of dual pseudo-potentials $\phi$ and $\phi^{*}$ parametrically depending on $\mathbf{f}$ and $\mathbf{v}$ (more precisely, on the difference $\mathbf{f}-\mathbf{f}^{n d}(\mathbf{v})$ ), starting from the knowledge of the elastic domain and the flow rule.

5.1. Non-associated flow rules. From now on, we assume to know the functions $f$ and $g$ that permit to define the constitutive law of the material. The dissipative forces are assumed to remain in a domain $\mathbb{E}$ defined by $f$ :

$$
\mathbb{E}:=\left\{\mathbf{f}^{d^{\prime}} \in \mathbb{V}^{*} / f\left(\mathbf{f}^{d^{\prime}}\right) \leq 0\right\}
$$

The function $f$ is differentiable and such that $f(\mathbf{0})=0$.

The flow $\dot{\mathbf{v}}$ is assumed to be defined on the boundary of $\mathbb{E}$ by the gradient of a differentiable function $g$ :

$$
\begin{aligned}
& \dot{\mathbf{v}}=\lambda \nabla g\left(\mathbf{f}^{d}\right) \\
& f\left(\mathbf{f}^{d}\right) \leq 0 \quad \lambda \geq 0, \quad \lambda f\left(\mathbf{f}^{d}\right)=0,
\end{aligned}
$$

If $\nabla g=\nabla f$, the law is associated, a normality condition of the type of (19) is fulfilled. Here, we are interested in the case where $\nabla g \neq \nabla f$. We want to construct a function $F$ depending on $f$ and $g$, permitting to retrieve the normality condition.

5.2. Construction of a state-dependent loading domain. We propose to define a state-dependent loading domain $\mathbb{E}_{\mathbf{y}}$ as follows

$$
\mathbb{E}_{\mathbf{y}}:=\left\{\mathbf{f}^{d^{\prime}} \in \mathbb{V}^{*} / F\left(\mathbf{f}^{d^{\prime}}, \mathbf{y}\right) \leq 0\right\}
$$

The loading function $F\left(\mathbf{f}^{d^{\prime}}, \mathbf{y}\right)$ is defined as the sum of the yield function $f\left(\mathbf{f}^{d^{\prime}}\right)$ and of a term, affine with respect to the variable $\mathbf{f}^{d^{\prime}}$, equal to zero when $\mathbf{f}^{d^{\prime}}=\mathbf{y}$, and depending on the gradient of the difference $g-f$ in $\mathbf{y}$ :

$$
F\left(\mathbf{f}^{d^{\prime}}, \mathbf{y}\right):=f\left(\mathbf{f}^{d^{\prime}}\right)+\nabla(g-f)(\mathbf{y}) \cdot\left(\mathbf{f}^{d^{\prime}}-\mathbf{y}\right)
$$


It can be noticed that for associated models, the gradient $\nabla(g-f)$ is null and one has:

$$
F\left(\mathbf{f}^{d^{\prime}}, \mathbf{y}\right):=f\left(\mathbf{f}^{d^{\prime}}\right)
$$

In this case, the loading function does not depend on $\mathbf{y}$ and is equal to $f$, i.e. $\mathbb{E}_{\mathbf{y}}=\mathbb{E}$.

Proposition 2. Let $f$ and $g$ be two convex and differentiable functions defined on $\mathbb{V}^{*}$. Then for any parameter $\mathbf{y} \in \mathbb{E}$ (i.e. $\mathbf{y} \in \mathbb{V}^{*}$ and $\left.f(\mathbf{y}) \leq 0\right)$ the domain $\mathbb{E}_{\mathbf{y}}$ defined by (23) with $F$ defined by (24) is non-empty, convex and the gradient of $F\left(\mathbf{f}^{d^{\prime}}, \mathbf{y}\right)$ with respect to $\mathbf{f}^{d^{\prime}}$ is

$$
\frac{\partial F}{\partial \mathbf{f}^{d^{\prime}}}\left(\mathbf{f}^{d^{\prime}}, \mathbf{y}\right)=\nabla g(\mathbf{y})
$$

Actually $\mathbb{E}_{\mathbf{y}}$ contains at least $\mathbf{y}$ since $F(\mathbf{y}, \mathbf{y})=f(\mathbf{y})$. And $F\left(\mathbf{f}^{d^{\prime}}, \mathbf{y}\right)$ is a convex function of $\mathbf{f}^{d^{\prime}}$, as it is the sum of the convex function $f\left(\mathbf{f}^{d^{\prime}}\right)$ and of a linear function of $\mathbf{f}^{d^{\prime}}$.

Corollary 1. Since the actual values of the thermodynamic forces fulfil $\mathbf{f}=\mathbf{f}^{n d}+\mathbf{f}^{d}$, then for $\mathbf{y}=\mathbf{f}-\mathbf{f}^{n d}=\mathbf{f}^{d}$, the following relationships are obtained:

$$
\left\{\begin{array}{l}
F\left(\mathbf{f}^{d}, \mathbf{f}-\mathbf{f}^{n d}\right)=f\left(\mathbf{f}^{d}\right) \\
\frac{\partial F}{\partial \mathbf{f}^{d^{\prime}}}\left(\mathbf{f}^{d}, \mathbf{f}-\mathbf{f}^{n d}\right)=\nabla g\left(\mathbf{f}^{d}\right)
\end{array}\right.
$$

Eq. (25) shows that if the domain $\mathbb{E}_{\mathbf{y}}$ varies in the space of dissipative forces $\mathbb{V}^{*}$ according to the variations of the parameter

$$
\mathbf{y}=\mathbf{f}-\mathbf{f}^{n d}=\mathbf{f}-\mathbf{f}^{n d}(\mathbf{v})
$$

then a normality condition with respect to the convex set $\mathbb{E}_{\mathbf{y}}$ is retrieved.

5.3. Pseudo-potentials and bipotentials. For any $\mathbf{y} \in \mathbb{E}$, the indicator function $\mathbb{I}_{\mathbb{E}_{\mathbf{y}}}\left(\mathbf{f}^{d^{\prime}}\right)$ of the state-dependent domain $\mathbb{E}_{\mathbf{y}}$ is a proper convex function for any $f$ proper and convex. As a result, its Legendre-Fenchel conjugate $\mathbb{I}_{\mathbb{E}_{\mathbf{y}}^{*}}^{*}\left(\dot{\mathbf{v}}^{\prime}\right)$ is a convex function of $\dot{\mathbf{v}}^{\prime}$. The pseudo potential $\phi\left(\dot{\mathbf{v}}^{\prime} ; \mathbf{y}\right)$ can be defined as:

$$
\left\{\begin{aligned}
\phi\left(\dot{\mathbf{v}}^{\prime} ; \mathbf{y}\right)= & \sup \left(\mathbf{f}^{d^{d^{\prime}}}\left(\mathbf{f}^{d^{\prime}} \cdot \dot{\mathbf{v}}^{\prime}-\mathbb{I}_{\mathbb{E}_{\mathbf{y}}}\left(\mathbf{f}^{d^{\prime}}\right)\right)\right. \\
& =\sup _{\forall \mathbf{f}^{d^{\prime}} \in \mathbb{E}_{\mathbf{y}}}\left(\mathbf{f}^{d^{\prime}} \cdot \dot{\mathbf{v}}^{\prime}\right)
\end{aligned}\right.
$$

and the conjugated pseudo-potential $\phi^{*}\left(\mathbf{f}^{d^{\prime}} ; \mathbf{y}\right)$ reads:

$$
\phi^{*}\left(\mathbf{f}^{d^{\prime}} ; \mathbf{y}\right)=\mathbb{I}_{\mathbb{E}_{\mathbf{y}}}\left(\mathbf{f}^{d^{\prime}}\right)
$$

For any $\mathbf{y} \in \mathbb{E}$, the function $\phi\left(\dot{\mathbf{v}}^{\prime}, \mathbf{y}\right)$ is convex and positively homogenous of degree one with respect to $\dot{\mathbf{v}}^{\prime}$ :

$$
\phi\left(\dot{\mathbf{v}}^{\prime} ; \mathbf{y}\right):=\phi^{* *}\left(\dot{\mathbf{v}}^{\prime} ; \mathbf{y}\right)
$$

By (26), for any $\mathbf{y} \in \mathbb{E}$ and for all $\mathbf{f}^{d^{\prime}} \in \mathbf{V}^{*}$ and all $\dot{\mathbf{v}}^{\prime} \in \mathbf{V}$, the Fenchel's inequality holds true:

$$
\phi^{*}\left(\mathbf{f}^{d^{\prime}} ; \mathbf{y}\right)+\phi\left(\dot{\mathbf{v}}^{\prime} ; \mathbf{y}\right) \geq \mathbf{f}^{d^{\prime}} \cdot \dot{\mathbf{v}}^{\prime}
$$


For any $\mathbf{y} \in \mathbb{E}$, the function $B\left(\mathbf{f}^{d^{\prime}}, \dot{\mathbf{v}}^{\prime} ; \mathbf{y}\right)$ defined as the sum:

$$
B\left(\mathbf{f}^{d^{\prime}}, \dot{\mathbf{v}}^{\prime} ; \mathbf{y}\right)=\phi^{*}\left(\mathbf{f}^{d^{\prime}} ; \mathbf{y}\right)+\phi\left(\dot{\mathbf{v}}^{\prime} ; \mathbf{y}\right)
$$

is convex with respect to $\mathbf{f}^{d^{\prime}}$ and to $\dot{\mathbf{v}}^{\prime}$ and fulfils:

$$
B\left(\mathbf{f}^{d^{\prime}}, \dot{\mathbf{v}}^{\prime} ; \mathbf{y}\right) \geq \mathbf{f}^{d^{\prime}} \cdot \dot{\mathbf{v}}^{\prime}
$$

Hence $B\left(\mathbf{f}^{d^{\prime}}, \dot{\mathbf{v}}^{\prime} ; \mathbf{y}\right)$ is a bipotential [7]. Equality in (29) or in (30) is obtained for $\dot{\mathbf{v}}_{\mathbf{y}}$ and for $\mathbf{f}_{\mathbf{y}}^{d}$ which are defined by:

$$
\dot{\mathbf{v}}_{\mathbf{y}} \in \partial_{\mathbf{f}^{d^{\prime}}} \phi^{*}\left(\mathbf{f}_{\mathbf{y}}^{d} ; \mathbf{y}\right), \quad \mathbf{f}_{\mathbf{y}}{ }^{d} \in \partial_{\dot{\mathbf{v}}^{\prime}} \phi\left(\dot{\mathbf{v}}_{\mathbf{y}} ; \mathbf{y}\right)
$$

If, in addition, we assume that, for any $\mathbf{y} \in \mathbb{E}$, the origin belongs to $\mathbb{E}_{\mathbf{y}}$, so that $\phi^{*}(\mathbf{0} ; \mathbf{y})=0$, and since $\phi^{*}$ is non-negative, then its conjugate is also non-negative so that we have $\phi^{* *}\left(\dot{\mathbf{v}}^{\prime}, \mathbf{y}\right) \geq 0$.

In summary, the resolution of the given mechanical problem can be obtained as follows. Starting from a known state of the material defined by $\mathbf{v}$, the nondissipative forces $\mathbf{f}^{n d}$ are calculated using the Helmoltz density of energy. Moreover, the forces $\mathbf{f}$ are known.

It follows that the quantity $\mathbf{f}-\mathbf{f}^{\text {nd }}$ is also known at each instant of the system evolution. Hence, if the parameter $\mathbf{y}$ is assumed to be equal to $\mathbf{f}-\mathbf{f}^{\text {nd }}$ at any instant, the admissible set $\mathbb{E}_{\mathbf{y}}$ (Eq. (23)) evolves during the system evolution. Then, using the normality property with respect to this set, the actual flow is defined using the first relationship in Eq. (31) and choosing $\mathbf{y}=\mathbf{f}-\mathbf{f}^{\text {nd }}$ :

$$
\dot{\mathbf{v}} \in \partial_{\mathbf{f}^{d^{\prime}}} \phi^{*}\left(\mathbf{f}^{d} ; \mathbf{y}\right)
$$

with $\mathbf{y}=\mathbf{f}-\mathbf{f}^{n d}$. This implies:

$$
\mathbf{f}^{d} \in \partial_{\dot{\mathbf{v}}^{\prime}} \phi(\dot{\mathbf{v}} ; \mathbf{y})
$$

Such definitions ensure that the condition given in Eq. (20) is fulfilled.

6. Conclusion. In some previous works, the authors have proposed statedependent pseudo-potentials for material models which do no belong to the family of Standard Generalized Materials: the endochronic theory [4], the Non-Linear Kinematic Hardening model [4] and the non-associated Drucker-Prager model [3]. Here, we give a general procedure to construct a pair of conjugate state-dependent pseudo-potentials. It can be applied not only to plasticity models, associated or not, but also to a large number of mechanical problems where the constitutive law implies that the dissipative thermodynamic forces remain in a given domain and with the flow of all the state variables not orthogonal to the boundary of this admissible domain. The introduction of a state-dependent loading function, and hence of a state-dependent admissible domain, in such a way that a normality property is recovered, permits then to construct a pair of pseudo-potentials which ensures that the second law of thermodynamics is fulfilled. The sum of these conjugated functions automatically defines a bipotential. 


\section{REFERENCES}

[1] J. J. Moreau, Sur les lois de frottement, de plasticité et de viscosité, Comptes Rendus de l'Académie des Sciences, Série II, 271 (1970), 608-611 (in French).

[2] B. Halphen and Q. S. Nguyen, Sur les matériaux standards généralisés, Journal de M écanique, 14 (1975), 39-63 (in French).

[3] S. Erlicher and N. Point, On the Associativity of the Drucker -Prager Model, VIII International Conference on Computational Plasticity - Fundamentals and Applications, Barcelona, Spain, 2005.

[4] S. Erlicher and N. Point, Endochronic theory, non-linear kinematic hardening rule and generalized plasticity: A new interpretation based on generalized normality assumption, International Journal of Solids and Structures, 43 (2006), 4175-4200.

[5] N. Point and S. Erlicher, Pseudo-potentials and loading surfaces for an endochronic plasticity theory with isotropic damage, Journal of Engineering Mechanics ASCE, 134 (2008), 832-842.

[6] N. Point and S. Erlicher, Pseudo-potentials and bipotential: A constructive procedure for non-associated plasticity and unilateral contact, Discret and Continuous Dynamical Systems - Series S, 6 (2013), 567-590.

[7] G. de Saxcé, A generalization of Fenchel's inequality and its applications to the constitutive laws, Comptes Rendus de Académie des Sciences, Série II, 314 (1992), 125-129.

[8] J. F. Babadjian, G. Francfort and M. G. Mora, Quasistatic evolution in non-associative plasticity: The cap model, SIAM J. Math. Anal, 44 (2012), 245-292.

[9] R. T. Rockafellar, Convex Analysis, Reprint of the 1970 original. Princeton Landmarks in Mathematics. Princeton Paperbacks. Princeton University Press, Princeton, NJ, 1997.

[10] H. Ziegler and C. Wehrli, The derivation of constitutive equations from the free energy and the dissipation function, Advances in Applied Mechanics, 25 (1987), 183-237.

[11] M. Jirásek and Z. P. Bazant, Inelastic Analysis of Structures, Wyley, Chichester, 2002.

[12] M. Frémond, Non-Smooth Thermomechanics, Springer-Verlag, Berlin, 2002.

[13] J. Lemaitre and J.-L. Chaboche, Mechanics of Solid Materials, Cambridge University Press, Cambridge, 1990.

E-mail address: nelly.point@enpc.fr

E-mail address: silvano.erlicher@egis.fr 